\title{
An interactive bi-objective shortest path approach: searching for unsupported nondominated solutions
}

\author{
J.M. Coutinho-Rodrigues ${ }^{\mathrm{a}, *}$, J.C.N. Clímaco ${ }^{\mathrm{b}}$, J.R. Current ${ }^{\mathrm{c}}$ \\ ${ }^{a}$ Department of Civil Engineering, F.C.T., University of Coimbra, 3049 Coimbra Codex, Portugal \\ ${ }^{\mathrm{b}}$ Faculty of Economics, University of Coimbra, 3000 Coimbra, Portugal \\ ${ }^{\mathrm{c}}$ Department of Management Sciences, The Ohio State University, 1775 College Avenue, Columbus, OH 43210, USA
}

Received August 1997; received in revised form October 1998

\begin{abstract}
In many network routing problems several conflicting objectives must be considered. Even for the biobjective shortest path problem, generating and presenting the whole set of nondominated solutions (paths) to a decision maker, in general, is not effective because the number of these paths can be very large. Interactive procedures are adequate to overcome these drawbacks. Current et al. [1] proposed an interactive approach based on a NISE-like procedure to search for nondominated supported solutions and using auxiliar constrained shortest path problems to carry out the search inside the duality gaps. In this paper we propose a new interactive approach to search for unsupported nondominated solutions (lying inside duality gaps) based on a $k$-shortest path procedure. Both approaches are compared. (C) 1999 Elsevier Science Ltd. All rights reserved.
\end{abstract}

\section{Scope and purpose}

Network routing problems are generally multidimensional in nature, and in many cases the explicit consideration of multiple objectives is adequate. Objectives related to cost, time, accessibility, environmental impact, reliability and risk are appropriated for selecting the most satisfactory ("best compromise") route in many problems. In general there is no single optimal solution in a multiobjective problem but rather, a set of nondominated solutions from which the decision maker must select the most satisfactory. However, generating and presenting the whole set of nondominated paths to a decision maker, in general, is not effective because the number of these paths can be very large. Interactive procedures are adequate to overcome these drawbacks. This paper introduces an interactive procedure to assist the decision maker in identifying the "best compromise" solution for the bi-objective shortest path problem. The procedure incorporates an efficient $k$-shortest path algorithm to identify nondominated solutions lying inside duality gaps. Test problem results indicate that the procedure can be readily executed on a PC for large-scale instances of problems.

Keywords: Shortest path; Multiple criteria; Network routing

*Corresponding author. Fax: 0035139822 511; e-mail: coutinho@dec.uc.pt. 


\section{Introduction}

Shortest path problems arise in a wide variety of practical problem settings, both as stand-alone models and as subproblems in more complex problem settings. The shortest path problem is quoted as the most common problem in combinatorial operations research [2] due to its numerous applications as the largest capacity path problem, the quickest path problem, the most reliable path problem, the minimum cost-time ratio problem, the minimum cost-reliability ratio problem and various routing problems. Ahuja et al. [3] describe applications in the telecommunications and transportation industries (whenever a message or a vehicle must be sent between two geographical locations as quickly or as cheaply as possible) and in urban traffic planning (urban planners use complex optimization models for computing traffic flow patterns based on shortest paths from origins to destinations). The wayfinding in emergency evacuations [4], the location of collective facilities (where the accessibility is a main concern) in an urban or regional context and the traffic assignment problem in a transportation network [5] are other practical examples which include the evaluation of shortest paths. Elimam et al. [6] present other civil engineering applications using shortest path-based models: the study of optimal sequences of wastewater treatment processes reducing pollutants levels at minimum cost to an acceptable standard (hydraulics) and the determination of minimum cost, energy efficient composite wall and roof structures (building structures). Consequently, many algorithmic approaches for location problems, vehicle routing, urban traffic engineering and even other problems that appear to have very different structures (as wastewater treatment or structures design) rely on the solution of shortest path problems.

Expressions as "cheap and quickest", "cheap and more reliable", "cheap with acceptable standard" or "cheap and energy efficient" come frequently associated with the shortest path problem - they suggest that models can be more realistic if more than one criterion is explicitly considered. In fact, it is well-recognized that many network routing problems are multiobjective in nature [7]. This has led to significant research effort devoted to formulating and solving multiobjective shortest path problems. The criteria, in addition to total path length (or cost), which have been addressed in these problems include: accessibility to the path $[8,9]$, travel time $[10,14]$ demand satisfaction [15], environmental protection [16], risk minimization [17], and reliability [18].

Efficient exact algorithms exist for the single objective shortest path problem [19]. Unfortunately, the multiobjective case (including the bi-objective case) is NP-complete [20]. Although the calculation of the whole set of the nondominated solutions in the bi-objective case can be done easily, it must be remarked that the number of the nondominated solutions can be very large. So, this is not, in general, an effective way of presenting alternative choices to a decision maker.

Note that in multiobjective shortest path problems the nondominated solutions are those paths where the values of the objective functions are such that it is not possible to find another feasible path better than the current one in at least one objective function without worsening the value of at least another objective.

Interactive procedures are adequate to overcome these drawbacks. Current et al. [1] proposed an interactive approach for the bi-objective shortest path problem. It starts by identifying a subset of the supported nondominated solutions using a NISE-like algorithm [21]. The search for unsupported nondominated solutions is carried out solving shortest path instances with additional linear constraints. Due to the computational complexity of the constrained shortest path problem 
Current et al. in [1] propose an interactive search of the previously chosen duality gap(s) based on the constrained shortest path algorithm of Handler and Zang [22]. To improve the computational efficiency of the approach, Clímaco et al. [23] and Coutinho-Rodrigues et al. [24] proposed a different related (to Current et al. [1]) approach using a $k$-shortest algorithm for searching nondominated solutions inside duality gaps.

In this paper we compare the two approaches mentioned above: computational results show that the $k$-shortest path-based approach is much faster than the constrained shortest path-based approach.

\section{Mathematical formulation and unsupported nondominated solutions searching procedure (GAPS)}

Several authors use a weighted sum of the objective functions (NISE-like or other approaches) to calculate supported nondominated solutions for the bi-objective shortest path problem. These can be identified by solving the following single-objective shortest path problem [SP], whose objective function is a convex combination of the original two objective functions, $Z_{1}$ and $Z_{2}$ [21, 25]. It must be emphasized that in many real-world applications the objective functions are conflituous. For instance, assume that the first objective $Z_{1}$, is to minimize route cost, and the second objective, $Z_{2}$, is to minimize total travel time. Points A, B, C, S and R in Fig. 1 represent the objective function values for five nondominated routes.

[SP] Minimize $\hat{Z}=w_{1} Z_{1}+w_{2} Z_{2}$

subject to

$$
\begin{aligned}
& \sum_{j \in N} X_{1 j}=1, \\
& \left.\sum_{i \in N} X_{i j}-\sum_{k \in N} X_{j k}=0 \quad \text { (for all } j \in N \mid j \neq 1, n\right), \\
& \sum_{i \in N} X_{i n}=1, \\
& X_{i j} \in(0,1), \text { for all } i, j \text { pairs, }
\end{aligned}
$$

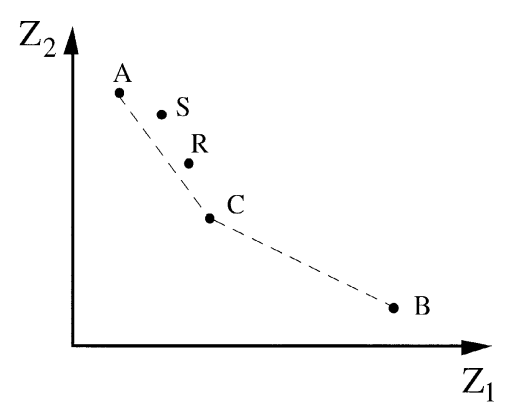

Fig. 1. A, B, C, R and $\mathrm{S}$ constitute the nondominated solutions set for a bi-objective $\left(Z_{1}, Z_{2}\right)$ shortest path problem. 
where

$$
\begin{aligned}
Z_{1} & =\sum_{i \in N} \sum_{j \in N} c_{i j} X_{i j}, \\
Z_{2} & =\sum_{i \in N} \sum_{j \in N} d_{i j} X_{i j}, \\
w_{1} & =\left|\frac{Z_{2 \alpha}-Z_{2 \beta}}{Z_{1 \alpha}-Z_{1 \beta}}\right|, \\
w_{2} & =1,
\end{aligned}
$$

$Z_{p \alpha}=$ the value of the $p$ th objective function for nondominated solution $\alpha$ ( $\alpha$ and $\beta$ are candidates to adjacent nondominated solutions)

$X_{i j}= \begin{cases}1 & \text { if arc } i, j \text { is on the path, } \\ 0 & \text { otherwise, }\end{cases}$

$c_{i j}, d_{i j}=$ nonnegative arc "costs",

$N=$ set of nodes in network,

node 1 is the source node,

node $n$ is the sink node,

$\alpha, \beta$ - supported nondominated solutions.

Unfortunately these procedures do not identify unsupported nondominated solutions such as routes corresponding to $\mathrm{S}$ and $\mathrm{R}[21,26]$.

In this paper we present a procedure to identify nondominated solutions in duality gaps (GAPS). "GAPS" incorporates a $k$-shortest algorithm, which, in the worst case, terminates when an upper bound is reached. The "GAPS" procedure is explained below. Computational results showing the efficiency of "GAPS" are presented in the next section.

"GAPS" is based upon solving a $k$-shortest path procedure [KSP] where $w_{1}$ was defined in (8) of [SP] and $\alpha$ and $\beta$ (here used) are true adjacent nondominated supported solutions of the original bi-objective shortest path problem (e.g. solutions B and C in Fig. 2). In this case "CPB" is the duality gap where new nondominated solutions must be searched. The point $\mathrm{P}$ (Fig. 2) and the values $Z_{1 \mathrm{P}}$ and $Z_{2 \mathrm{P}}$ according to Eq. (10) enable us to obtain the first upper bound for the $k$-shortest search (i.e. $w_{1} Z_{1 \mathrm{P}}+w_{2} Z_{2 \mathrm{P}}$ ) in the duality gap defined by the points $\mathrm{B}, \mathrm{C}$ and $\mathrm{P}$. Note that $Z_{1 \mathrm{P}}=Z_{1 \mathrm{~B}}$ and that $Z_{2 \mathrm{P}}=Z_{2 \mathrm{C}}$ (for details see Current et al. [1] or Cohon [21]).

$w_{1}$ and $w_{2}$ were defined in order to obtain $\hat{Z}$ [in (SP)] such that the line passing though $\overline{\mathrm{BC}}$ is a constant cost line for $\hat{Z}$. The optimal solution is obtained for $\mathrm{B}$ and $\mathrm{C}$. The $k$-shortest path algorithm starts by looking for the first solution inside the duality gap ("CPB"). Suppose it is D in Fig. 3.

Note that to identify solutions inside the duality gap the solutions obtained using the $k$-shortest algorithm must verify the upper bounds of the duality gap. The first solution obtained in the duality gap is nondominated (by definition of the nondominated solutions).

By definition of nondominated solutions new eventual nondominated solutions inside the duality gap must be in the shadow area of Fig. 4. An improved upper bound for $\hat{Z}$ can be obtained from $\mathrm{P}^{\prime}$ in Fig. 4. It is clear how to continue the search step by step in the duality gap. It may stop 


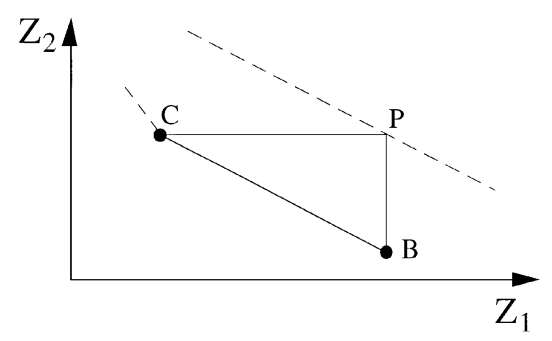

Fig. 2. The duality gap "CPB”.

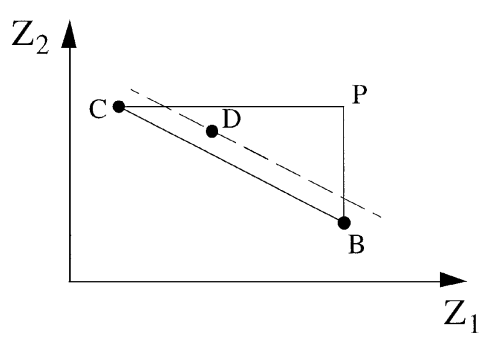

Fig. 3. The first solution, D, inside the duality gap.

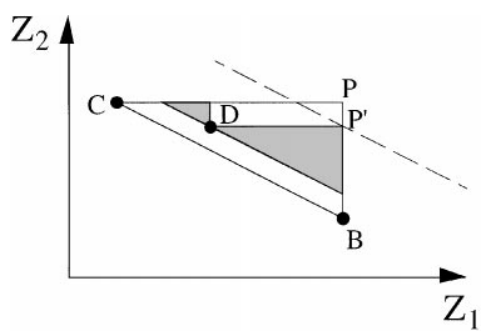

Fig. 4. Upper bound for $\hat{Z}$ and area for eventual nondominated solutions.

when no more nondominated solutions exist inside the duality gap or alternatively where the decision maker decides to stop because she/he is satisfied.

The decision support tool we developed enables a graphical representation of the gap (see Coutinho-Rodrigues et al. [24]) with the identification of the nondominated solutions and of the shadow areas corresponding to each state of the search. So, an interactive search inside the gap is available alternatively with the automatic calculation of the whole set of nondominated solutions inside the gap.

\section{Computational results}

To check the efficiency of "GAPS" 39 randomly generated networks were tested. These networks were generated considering that every pair of nodes in the network is connected by at least one 
Table 1

Summary of test problem results

\begin{tabular}{|c|c|c|c|c|c|c|c|c|c|c|c|}
\hline \multirow[t]{4}{*}{ Prob \# } & \multicolumn{2}{|c|}{$\begin{array}{l}\text { Network } \\
\text { dimensions }\end{array}$} & \multirow{4}{*}{$\begin{array}{l}\text { \# Non- } \\
\text { dominated } \\
\text { Solutions } \\
\text { inside } \\
\text { the Gap }\end{array}$} & \multicolumn{3}{|c|}{$\begin{array}{l}\text { Total times } \\
\text { searching the gap }\end{array}$} & \multicolumn{3}{|c|}{$\begin{array}{l}\text { Ratios of total search } \\
\text { times in the gap }\end{array}$} & \multirow{2}{*}{\multicolumn{2}{|c|}{$\begin{array}{l}\text { Search times for } \\
\text { the best solutions } \\
\text { optimizing } \hat{Z}\end{array}$}} \\
\hline & \multirow{3}{*}{ \# Nodes } & \multirow{3}{*}{ \# Arcs } & & \multirow{3}{*}{$\begin{array}{l}\mathrm{AlgA} \\
(\mathrm{sec})\end{array}$} & \multirow{3}{*}{$\begin{array}{l}\mathrm{AlgD} \\
(\mathrm{sec})\end{array}$} & \multirow{3}{*}{$\begin{array}{l}\text { AlgC } \\
(\mathrm{sec})\end{array}$} & \multirow{3}{*}{$\frac{\mathrm{Alg} \mathrm{D}}{\mathrm{A} \lg \mathrm{A}}$} & \multirow{3}{*}{$\frac{\mathrm{AlgD}}{\mathrm{AlgD}}$} & \multirow{3}{*}{$\frac{\mathrm{Alg} C}{\mathrm{Alg} A}$} & & \\
\hline & & & & & & & & & & $\mathrm{Alg} \mathrm{A}$ & $\mathrm{AlgD}$ \\
\hline & & & & & & & & & & $(\mathrm{sec})$ & $(\mathrm{sec})$ \\
\hline 1 & 200 & 800 & 4 & 0.47 & 1.32 & 11.68 & 2.8 & 8.9 & 25.0 & 0.38 & 0.33 \\
\hline 2 & 250 & 800 & 3 & 0.75 & 2.50 & 18.58 & 3.3 & 7.4 & 24.8 & 0.58 & 0.13 \\
\hline 3 & 250 & 800 & 4 & 0.63 & 1.25 & 15.72 & 2.0 & 12.6 & 24.8 & 0.53 & 0.15 \\
\hline 4 & 250 & 800 & 2 & 0.62 & 2.15 & 12.43 & 3.5 & 5.8 & 20.2 & 0.55 & 0.50 \\
\hline 5 & 275 & 1000 & 3 & 0.70 & 2.72 & 18.17 & 3.9 & 6.7 & 26.0 & 0.65 & 0.60 \\
\hline 6 & 275 & 1000 & 5 & 0.70 & 1.85 & 27.75 & 2.6 & 15.0 & 39.6 & 0.65 & 0.58 \\
\hline 7 & 275 & 1000 & 4 & 0.72 & 2.75 & 24.93 & 3.8 & 9.1 & 34.8 & 0.63 & 0.17 \\
\hline 8 & 275 & 1000 & 4 & 0.65 & 1.82 & 18.62 & 2.8 & 10.2 & 28.6 & 0.62 & 0.60 \\
\hline 9 & 300 & 800 & 4 & 0.92 & 1.90 & 26.63 & 2.1 & 14.0 & 29.1 & 0.82 & 0.15 \\
\hline 10 & 300 & 800 & 4 & 0.77 & 1.88 & 24.25 & 2.5 & 12.9 & 31.6 & 0.75 & 0.68 \\
\hline 11 & 300 & 1000 & 4 & 0.75 & 1.57 & 21.43 & 2.1 & 13.7 & 28.6 & 0.72 & 0.18 \\
\hline 12 & 300 & 1000 & 6 & 0.85 & 3.10 & 41.23 & 3.6 & 13.3 & 48.5 & 0.75 & 0.68 \\
\hline 13 & 300 & 1000 & 4 & 0.78 & 1.82 & 21.13 & 2.3 & 11.6 & 27.0 & 0.70 & 0.68 \\
\hline 14 & 300 & 1000 & 6 & 0.93 & 3.85 & 40.22 & 4.1 & 10.4 & 43.1 & 0.75 & 0.70 \\
\hline 15 & 300 & 1000 & 3 & 0.85 & 2.50 & 22.87 & 2.9 & 9.1 & 26.9 & 0.75 & 0.68 \\
\hline 16 & 400 & 1000 & 5 & 1.28 & 2.70 & 39.63 & 2.1 & 14.7 & 30.9 & 1.22 & 1.17 \\
\hline 17 & 400 & 1000 & 2 & 1.25 & 0.83 & 18.28 & 0.7 & 21.9 & 14.6 & 1.25 & 0.20 \\
\hline 18 & 400 & 1000 & 4 & 1.32 & 2.72 & 34.57 & 2.1 & 12.7 & 26.3 & 1.25 & 1.17 \\
\hline 19 & 400 & 1200 & 6 & 1.25 & 3.15 & 48.15 & 2.5 & 15.3 & 38.5 & 1.20 & 1.18 \\
\hline 20 & 400 & 1200 & 4 & 1.37 & 3.90 & 43.03 & 2.9 & 11.0 & 31.5 & 1.28 & 1.17 \\
\hline 21 & 400 & 1200 & 3 & 1.23 & 3.10 & 27.30 & 2.5 & 8.8 & 22.1 & 1.22 & 1.18 \\
\hline 22 & 500 & 1300 & 4 & 1.90 & 3.18 & 47.35 & 1.7 & 14.9 & 24.9 & 1.90 & 1.80 \\
\hline 23 & 500 & 1300 & 5 & 1.97 & 4.05 & 74.37 & 2.1 & 18.4 & 37.8 & 1.92 & 1.78 \\
\hline 24 & 500 & 1300 & 4 & 1.93 & 3.80 & 48.47 & 2.0 & 12.8 & 25.1 & 1.87 & 1.80 \\
\hline 25 & 1000 & 6000 & 4 & 7.20 & 11.58 & 181.28 & 1.6 & 15.7 & 25.2 & 7.15 & 6.98 \\
\hline 26 & 1000 & 6000 & 8 & 7.82 & 58.27 & 528.85 & 7.5 & 9.1 & 67.7 & 7.25 & 7.00 \\
\hline 27 & 1000 & 6000 & 6 & 7.40 & 15.08 & 342.43 & 2.0 & 22.7 & 46.3 & 7.20 & 0.88 \\
\hline 28 & 1000 & 6000 & 5 & 7.27 & 18.77 & 242.60 & 2.6 & 12.9 & 33.4 & 7.10 & 6.98 \\
\hline 29 & 1000 & 6000 & 6 & 7.47 & 27.15 & 323.87 & 3.6 & 11.9 & 43.4 & 7.12 & 0.88 \\
\hline 30 & 2000 & 8000 & 4 & 27.58 & 39.77 & 817.93 & 1.4 & 20.6 & 29.7 & 27.47 & 26.55 \\
\hline 31 & 2000 & 8000 & 3 & 27.45 & 32.13 & 642.75 & 1.2 & 20.0 & 23.4 & 27.42 & 26.55 \\
\hline 32 & 2000 & 8000 & 4 & 27.52 & 43.52 & 848.77 & 1.6 & 19.5 & 30.8 & 27.42 & 26.55 \\
\hline 33 & 2000 & 8000 & 3 & 27.57 & 43.17 & 669.02 & 1.6 & 15.5 & 24.3 & 27.43 & 26.55 \\
\hline 34 & 2000 & 8000 & 4 & 27.35 & 42.55 & 693.90 & 1.6 & 16.3 & 25.4 & 27.23 & 27.63 \\
\hline 35 & 4000 & 8000 & 3 & 108.10 & 120.08 & 2005.25 & 1.1 & 16.7 & 18.5 & 108.03 & 105.38 \\
\hline 36 & 4000 & 8000 & 3 & 109.17 & 128.43 & 2509.52 & 1.2 & 19.5 & 23.0 & 109.08 & 105.40 \\
\hline 37 & 4000 & 8000 & 2 & 108.52 & 118.92 & 1510.12 & 1.1 & 12.7 & 13.9 & 108.47 & 105.27 \\
\hline 38 & 6000 & 8000 & 3 & 242.07 & 257.18 & 4471.03 & 1.1 & 17.4 & 18.5 & 242.00 & 236.40 \\
\hline 39 & 6000 & 8000 & 2 & 243.02 & 248.62 & 3334.52 & 1.0 & 13.4 & 13.7 & 243.00 & 236.37 \\
\hline
\end{tabular}

AlgA - Algorithm with Azevedo et al. [28-30] " $k$-shortest".

AlgD - Algorithm with Dreyfus [27] " $k$-shortest".

AlgC - Algorithm of Current et al. [1] with dual using lagrangean relaxation. 
path. This condition seems realistic for many real-world applications such as routing problems in transportation and communication systems. Networks of various sizes and densities were generated. Networks from 200 nodes and 800 arcs to 6000 nodes and 8000 arcs were used. Arc node ratios vary from 1.35 to 6 . The whole set of the nondominated solutions was identified in one "ad hoc" chosen gap for each test network. All tests were performed on a Macintosh Ci computer with a 68030 Motorola CPU and a math coprocessor.

The GAPS procedure was tested with two $k$-shortest path algorithms: Dreyfus [27] and Azevedo et al. [28-30]. The Dreyfus algorithm was encoded according to the suggestions of Lawler [31]. They are referred to as $\mathrm{AlgD}$ and $\mathrm{AlgA}$, respectively. In addition, all the nondominated solutions were identified in the selected gaps using the constrained shortest path algorithm of Handler and Zang [22] as suggested by Current et al. [1]. This approach is referred to as AlgC.

The results of these tests are presented in Table 1. Column 1 identifies the problem and columns 2 and 3 list the number of nodes and arcs, respectively. Column 4 gives the number of nondominated solutions within the searched gap. Columns 5-7 report (in CPU seconds) the total time required to search inside the gap via $\mathrm{Alg} A, \mathrm{AlgD}$ and $\mathrm{AlgC}$, respectively. Columns 8-10 report the ratios of total search time for $\mathrm{AlgD}$ : $\mathrm{Alg} A ; \mathrm{AlgC}: \mathrm{AlgD}$; and $\mathrm{AlgC}$ : $\mathrm{Alg} \mathrm{A}$, respectively. Columns 11 and 12 report (in CPU seconds) the time to calculate the best solution of [SP] using AlgA and $\mathrm{AlgD}$.

The ratios in columns 8-10 indicate that the $k$-shortest path algorithms (AlgA and $\mathrm{AlgD}$ ) were clearly more efficient than the constrained shortest path algorithm ( $\mathrm{AlgC}$ ). $\mathrm{AlgD}$ required from $1 / 5$ to $1 / 23$ of the time required by $\mathrm{AlgC}$ and $\mathrm{Alg}$ A requiring from $1 / 13$ to $1 / 68$ of the time required by $\mathrm{A} \operatorname{lgC}$, to identify all the nondominated solutions in the gap. AlgA was significantly more efficient than $\mathrm{AlgD}$ in solving 38 of the 39 test problems.

In the last two columns of Table 2 we present the run times necessary to identify the first solution inside the gap for Alg A and AlgD. The comparison of those columns with columns 5 and 6 show that AlgA was particularly efficient in identifying gap solutions from the second best. In order to emphasize the comparison between AlgA and AlgC, Figs. 5 (ratios of total search times inside the gap) and 6 (ratios of average searching times from the second best) are presented.

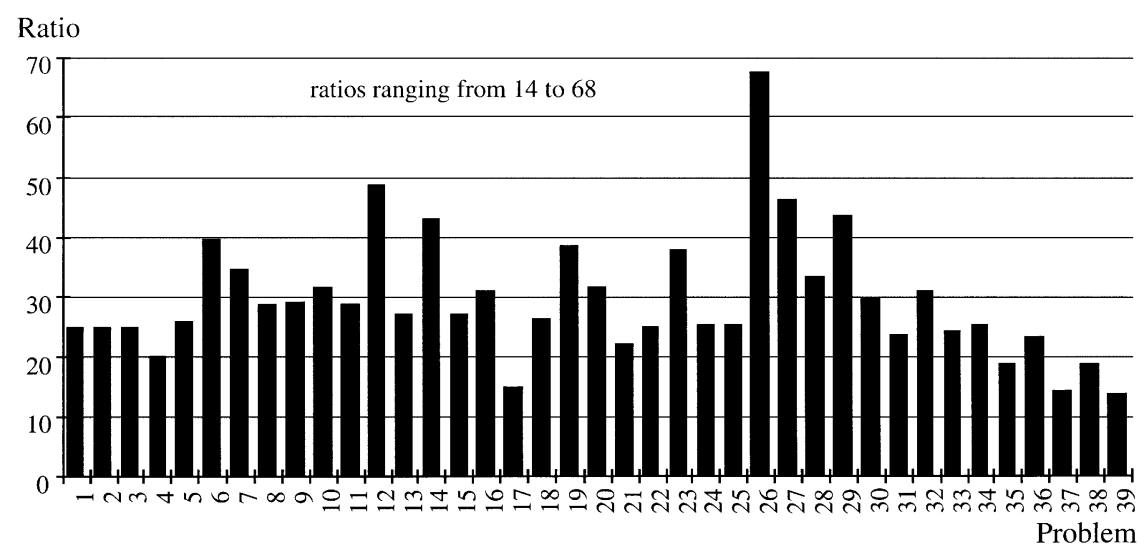

Fig. 5. Rations $\mathrm{A} \operatorname{lgC} / \mathrm{Alg} \mathrm{A}$ of total search times inside the gap for the test problems. 


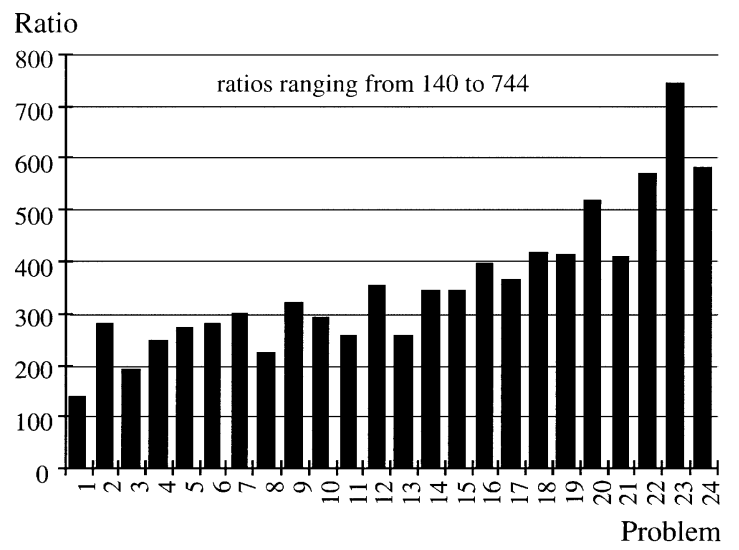

Problems 1-24

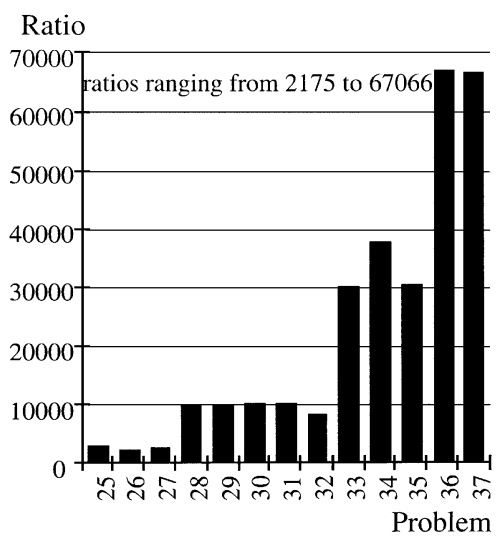

Problems 25-39

Fig. 6. Rations $\mathrm{AlgC} / \mathrm{A} \lg \mathrm{A}$ : average searching times for identifying an additional nondominated solution inside the duality gap.

\section{Conclusions}

In this paper we compare two approaches for searching nondominated solutions inside duality gaps concerning bi-objective shortest path problems.

Two major points must be emphasized from the results:

- The approach using a $k$-shortest path algorithm is unquestionably faster than the approach using a constrained shortest path algorithm.

- Although our implementation of the algorithm by Azevedo et al. [28-30] does not take in account the most recent computational improvements proposed by its authors, it works in our problem clearly better than the $k$-shortest path algorithm by Dreyfus [27].

\section{References}

[1] Current JR, Revelle CS, Cohon JL. An interactive approach to identify the best compromise solution for two objective shortest path problems. Computers \& Operations Research 1990;17:197-8.

[2] Chen Y, Chin Y. The quickest path problem. Computers and Operations Research 1990;17:153-61.

[3] Ahuja R, Magnanti T, Orlin J. Network flows. Englewood Cliffs, NJ: Prentice-Hall, 1993.

[4] Løvås G. Models of wayfinding in emergency evacuations. European Journal of Operational Research 1998;105:371-89.

[5] Larson R, Odoni A. Urban operations research. Englewoods Cliffs, NJ: Prentice-Hall, 1981.

[6] Elimam A, Kohler D. Two engineering applications of a constrained shortest-path model. European Journal of Operational Research 1997;103:426-38.

[7] Current J, Marsh M. Multiobjective transportation network design and routing problems: taxonomy and annotation. European Journal of Operational Research 1993;65:1-15.

[8] Halder DK, Majumder A. A method for selecting optimum number or stations for a rapid transit line: an application in Calcutta tube rail. In: Jaiswal NK, editors, Scientilic management of transport systems. Amsterdam: NorthHolland, 1981:97-108. 
[9] Current JR, ReVelle CS, Cohon JL. The median shortest path problem: a multiobjective approach to analyze cost vs. accessibility in the design or transportation networks. Transportation Science 1987;21:490-503.

[10] Aneja YP, Nair KPK. Bicriteria transportation problem. Management Science 1979;25:73-8.

[11] Dial R. A model and algorithm for multicriteria route-mode choice. Transportation Research 1979;13B:311-6.

[12] Dallenbach HG, Dekluyver CA. Note on multiple objective dynamic programming. Journal of the Operational Research Society 1980;31:591-4.

[13] Clímaco JCN, Martins EQ. A Bicriteria shortest path algorithm. European Journal of Operational Research 1982;2:399-404.

[14] Martins EQ. On a multi-criteria shortest path problem. European Journal of Operational Research 1984; $16: 236-45$.

[15] Current JR, ReVelle CS, Cohon JL. The maximum covering/shortest path problem: a multiobjective network design and routing formulation. European Journal of Operational Research 1985;21:189-99.

[16] Engberg D, Cohon J, ReVelle C. Multiobjective siting of a natural gas pipeline, Proceedings of the Eleventh Annual Pittsburgh Conference on Modeling and Simulation 11. Instrument Society of America, Research Triangle Park, N.C., 1983.

[17] Current JR, ReVelle CS, Cohon JL. The minimum-covering/shortest-path problem. Decision Science 1988;19: 490-503.

[18] Hansen P. Bicriteria path problems. In: Beckmann M, Kunzi HP, editors, Lecture Notes in Economics and Mathematical Systems vol. 177. Springer, Berlin 1980:109-27.

[19] Deo N, Pang C. Shortest-path algorithms: taxonomy and annotation. Networks 1984;14:273-323.

[20] Garey M, Johnson D. Computers and intractibility: A Guide to the Theory of NP-Completeness. San Francisco, CA: Freeman, 1979.

[21] Cohon J. Multiobjective programming and planning. New York: Academic Press, 1978.

[22] Handler GY, Zang I. A dual algorithm for the constrained shortest path problem. Networks 1980;10:293-310.

[23] Clímaco J, Coutinho-Rodrigues J. On an interactive bicriteria shortest path algorithm, First International Workshop on Project Management and Scheduling, Lisbon, July 1988.

[24] Coutinho-Rodrigues J, Clímaco J, Current J. A PC-based interactive decision support system for two objective direct delivery problems. Journal of Business Logistics 1994;15(1):305-22.

[25] White DJ. The set of efficient solutions for multiple objective shortest path problems. Computers and Operations Research 1982;9:101-7.

[26] Henig IH. The shortest path problem with two objective functions. European Journal of Operational Research 1985;25:281-91.

[27] Dreyfus. An appraisal of some shortest path algorithms. Operations Research 1969;17:395-412.

[28] Azevedo JA, Madeira J, Martins EQV, Martins F. A shortest paths ranking algorithm. AIRO'90-Proceedings of the Annual Conference, Operational Research Society of Italy, 1990;1001-11.

[29] Azevedo JA, Costa M, Madeira J, Martins EQV. An algorithm for the ranking of shortest paths. European Journal of Operational Research 1993;69:97-106.

[30] Azevedo JA, Madeira J, Costa M, Martins EQV, Pires F. A computational improvement for a shortest paths ranking algorithm. European Journal of Operational Research 1994;73:188-91.

[31] Lawler EL. Combinatorial optimization: networks and matroids. New York: Holt Rinehart and Winston, 1969.

João Coutinho-Rodrigues is currently Professor at the Department of Civil Engineering, Faculty of Science and Technology, University of Coimbra, and senior researcher in the Decision Support Systems group at INESC-Coimbra. He received his Degree in Civil Engineering, his M.Sc. in Computer Science and Ph.D. in Civil Engineering from the University of Coimbra. Research interests include: decision support systems, multicriteria analysis, networks, GIS, and applications in urban engineering, transportation planning and environmental engineering.

João C. N. Clímaco is a full professor at the Faculty of Economies, University of Coimbra and a researcher at the DSS group of INESC. He received a degree in Electrical Engineering from the University of Oporto, a M.Sc. degree in Control Systems from the Imperial College of Science and Technology, University of London, and a Ph.D. in Electrical Engineering (Optimization and Systems Theory) from the University of Coimbra. He also received the Diploma of 
Membership of the Imperial College of Science and Technology. Dr. Clímaco"s principal research interests are in multiple criteria decision making, mathematical programming, decision support systems, telecommunications network planning and energy planning. He is member of the editorial board of the Journal of Group Decisions and Negotiation.

John R. Current is a member of the Faculty of Management Sciences at The Ohio State University and has been a visiting Professor at the Faculty of Economics, University of Coimbra. He received a BA in political science from DePauw University and an M.S. in urban planning and a Ph.D. in Geography and environmental engineering from the John Hopkins University. Dr. Current's primary research interests are in the areas of multiobjective programming, location analysis, and network design. He is an editor of the journal Location Science. 